\title{
A Feasible Control Method for Incomplete Symmetrical Marine Robot
}

\author{
Lei Zhang, ${ }^{1, *}$, Zaopeng Dong ${ }^{2}$, Yongsuo Yan ${ }^{1}$ and Yuyin Cao ${ }^{1}$ \\ ${ }^{1}$ Harbin Engineering University, College of Shipbuilding Engineering, Harbin City, HelongJiang Province, China \\ ${ }^{2}$ Wuhan University of Technology, School of Transportation, Wuhan City, Hubei Province, China \\ ${ }^{*}$ Corresponding author
}

\begin{abstract}
For the incomplete symmetrical underactuated USV (unmanned surface vehicle), this paper proposed a global asymptotic stabilization control method. The global diffeomorphism transformation for the USV model was performed twice in order to obtain the form of nonlinear cascade systems, so the stabilization problem of the original USV system was simplified to the stabilization of the cascade systems. Then the cascade system stabilization controller was designed based on Backstepping, realize the global asymptotic stabilization control of the incomplete symmetrical underactuated USV. Effectiveness and reliability of the research methods and the controller designed was vertified by simulation results.
\end{abstract}

Keywords-USV; global asymptotic stabilization; underactuated control; cascade systems; backstepping

\section{INTRODUCTION}

Surface robots, also called USV (unmanned surface vehicle), was a kind of small ships, which can autonomous navigate in the actual ocean and complete corresponding mission. Because of the superiority in intelligence, surveillance, reconnaissance, naval protection, demining, antisubmarine, precision, search and rescue, navigation and hydrographic investigation, USV had been paid much attention by the world navy, USV was developing rapidly in recent decades. The motion control technology was a key technology to realize unmanned autonomous control of USV, and the stabilization control was a key link. About the stabilization control of USV, many researchers had conducted relative study, and published a number of research results.

M.Reyhanoglu (1996) discussed the stabilization of USV control. In this work, transformation method was used to design the time invariant continuous feedback control law, make the system to achieve global exponential stability. Base on differential geometry theory, J.Ghomman et al. studied the stabilization control of underactuated USV and designed time-varying discrete feedback control law using differential homeomorphism transform. Through adding index convergence item to the state feedback control of underactuated ships, Liao et al. designed the time-varying smooth stabilization control law, and make the system to achieve global exponential stability. Ma Baoli got the exponential stability variable switching control law of underactuated USV using discontinuous coordinate transformation and Lyapunov like switching function, but because of the switching, the control law is discontinuous. LIU et al. proposed an algorithm using diffeomorphism transformation and control input transformation, transformed the motion model of ship into a cascade system, designed feedback control law based on Backstepping, and demonstrate the stability. Kristin and Thor proposed the feedback control law about time function using combined back-to-back method and homogenous method, accelerated the speed of system stabilization, and verified the system clam in a field near the equilibrium point by experimental, but there was an ineradicable oscillations at the equilibrium point. Mazenc et al designed a time-varying smooth state feedback controller using Backstepping, made the USV can achieve uniform global asymptotic stability.

Except for some special limitations and defects, all of the stabilization controllers of USV designed by the scholars, were assume that the motion model of USV was fore-aft and bilateral symmetry (because just study the USV motion in the horizontal plane, the up-down symmetry was not considered). This did not tally with the actual model of USV, USV was only bilateral symmetrical, but not fore-aft symmetrical. This paper studied the incomplete symmetrical USV, the USV was bilateral symmetrical, but not fore-aft symmetrical, in this case, nonzero terms existed in the off-diagonal elements of the USV added mass coefficient, the inertia coefficient matrix and the damping coefficient matrix of the USV motion were no longer the diagonal line of the standard, not all the off-diagonal elements were zero.

\section{INCOMPLETE SYMMETRICAL USV MODEL}

\section{A. Kinematics and Dynamics Model}

This paper mainly study the motion of USV in surge, sway and yaw, the kinematic model of the system can be written as follows[8]:

$$
\dot{\eta}=R(\varphi) v
$$

where, $\quad \eta$ is the state vector of systems, and $\eta=[x, y, \varphi]^{\mathrm{T}}$. $x$ is the surge displacement, $y$ is the sway displacement, $\varphi$ is the yaw angle, the reference direction is north. $R$ is Rotation Matrix of yaw, defined as: $R(\varphi)=\left(\begin{array}{ccc}\cos \varphi & -\sin \varphi & 0 \\ \sin \varphi & \cos \varphi & 0 \\ 0 & 0 & 1\end{array}\right)$. 
where, $v$ is the velocity vector, and $v=[u, v, r]^{\mathrm{T}} . u$ is the surge velocity of the USV, $v$ is the sway velocity, $r$ is the yaw palstance.

The nonlinear dynamical model of the USV in horizontal plane is as follow[8]:

$$
M(v) \dot{v}+C(v) v+D(v) v=\tau
$$

where, $M$ is the inertia coefficient matrix, $C$ is the centripetal force matrix, $D$ is the damping coefficient matrix. They are respectively defined as:

$$
\begin{gathered}
M=\left[\begin{array}{ccc}
m_{11} & 0 & 0 \\
0 & m_{22} & m_{23} \\
0 & m_{32} & m_{33}
\end{array}\right], \quad C=\left[\begin{array}{ccc}
0 & 0 & C_{13} \\
0 & 0 & C_{23} \\
C_{31} & C_{32} & 0
\end{array}\right], \quad D=\left[\begin{array}{ccc}
d_{11} & 0 & 0 \\
0 & d_{22} & d_{23} \\
0 & d_{32} & d_{33}
\end{array}\right], \\
C_{13}=-C_{13}=-m_{22} v-\frac{1}{2}\left(m_{23}+m_{32}\right) r \quad C_{23}=-C_{32}=m_{11} u .
\end{gathered}
$$

where, $\tau$ is control force, the USV system is underactuated, so just consider the input in surge and yaw, and $\tau=\left[\begin{array}{lll}\tau_{u} & 0 & \tau_{r}\end{array}\right]^{\mathrm{T}}$.

\section{B. Global Diffeomorphism Transformation}

Due to the off-diagonal elements of the matrix (the inertia coefficient matrix and the damping coefficient matrix) of the USV system are not all zero, the analysis and design of the USV system are more difficult. In order to overcome this problem, the global diffeomorphism transformation for the USV model was performed twice, and obtained the form of cascade systems.

1) Diffeomorphism transformation-A

Define $\left\{\begin{array}{l}z_{1}^{*}=x+\frac{m_{23}}{m_{22}}(\cos \varphi-1) \\ z_{2}^{*}=y+\frac{m_{23}}{m_{22}} \sin \varphi \\ z_{3}^{*}=\varphi \\ z_{4}^{*}=u \\ z_{5}^{*}=v+\frac{m_{23}}{m_{22}} r \\ z_{6}^{*}=r\end{array}\right.$, it can be described as:

$$
\left\{\begin{array}{l}
\dot{z}_{1}^{*}=z_{4}^{*} \cos \left(z_{3}^{*}\right)-z_{5}^{*} \sin \left(z_{3}^{*}\right) \\
\dot{z}_{2}^{*}=z_{4}^{*} \sin \left(z_{3}^{*}\right)+z_{5}^{*} \cos \left(z_{3}^{*}\right) \\
\dot{z}_{3}^{*}=z_{6}^{*} \\
\dot{z}_{4}^{*}=f_{1}^{*} \\
\dot{z}_{5}^{*}=-\alpha z_{4}^{*} z_{6}^{*}-\beta z_{5}^{*}+\gamma z_{6}^{*} \\
\dot{z}_{6}^{*}=f_{2}^{*}
\end{array}\right.
$$

If the control input by feedback can be changed as:

$$
\left\{\begin{aligned}
f_{1}^{*}= & \frac{1}{m_{11}}\left(\tau_{u}+m_{22} v r+\frac{\left(m_{23}+m_{32}\right)}{2} r^{2}-d_{11} u\right) \\
f_{2}^{*}= & \frac{m_{22}}{m_{22} m_{33}-m_{32} m_{23}}\left\{\tau_{r}+\frac{\left(m_{11} m_{22}-m_{22}^{2}\right) u v+\left[m_{11} m_{32}-\frac{m_{22}\left(m_{23}+m_{32}\right)}{2}\right] u r}{m_{22}}\right. \\
& \left.+\frac{\left(m_{32} d_{22}-m_{22} d_{32}\right) v-\left(m_{22} d_{33}-m_{32} d_{23}\right) r}{m_{22}}\right\}
\end{aligned}\right.
$$

where, $\quad \alpha=\frac{m_{11}}{m_{22}}, \quad \beta=\frac{d_{22}}{m_{22}}, \quad \gamma=\frac{d_{22} m_{23}}{m_{22}^{2}}-\frac{d_{23}}{m_{22}}$.

The stabilization target of the original USV system is: design suitable control input $\tau_{u}, \tau_{r}$, make $u, v, r, x, y, \varphi$ of the USV system global asymptotic stabilization. After the diffeomorphism transformation, the target is: design suitable control input $f_{1}^{*}, f_{2}^{*}$, make $z_{1}^{*}, z_{2}^{*}, z_{3}^{*}, z_{4}^{*}, z_{5}^{*}, z_{6}^{*}$ global asymptotic stabilization. Because the system is still complex after transformation, not suitable for the stability analysis of stabilization control and the design of controller directly, the global diffeomorphism transformation for System(3) was performed again.

2) Diffeomorphism transformation-B

Define: $\left\{\begin{array}{l}z_{1}=z_{1}^{*} \cos \left(z_{3}^{*}\right)+z_{2}^{*} \sin \left(z_{3}^{*}\right) \\ z_{2}=-z_{1}^{*} \sin \left(z_{3}^{*}\right)+z_{2}^{*} \cos \left(z_{3}^{*}\right)+\frac{1}{\beta} z_{5}^{*}-\frac{\gamma}{\beta} z_{3}^{*} \\ z_{3}=z_{3}^{*} \\ z_{4}=-\frac{\alpha}{\beta} z_{4}^{*}-z_{1}^{*} \cos \left(z_{3}^{*}\right)-z_{2}^{*} \sin \left(z_{3}^{*}\right) \\ z_{5}=z_{5}^{*} \\ z_{6}=z_{6}^{*}\end{array}\right.$.

Transform the control input by feedback change as:

$$
\left\{\begin{array}{l}
f_{1}=-\frac{\alpha}{\beta} f_{1}^{*}+\frac{\beta}{\alpha}\left(z_{1}+z_{4}\right)-z_{2} z_{6}+\frac{1}{\beta} z_{5} z_{6}-\frac{\gamma}{\beta} z_{3} z_{6} \\
f_{2}=f_{2}^{*}
\end{array}\right.
$$

Then, obtain the new system as follow:

$$
\left\{\begin{array}{l}
\dot{z}_{1}=-\frac{\beta}{\alpha} z_{1}-\frac{\beta}{\alpha} z_{4}+z_{2} z_{6}-\frac{1}{\beta} z_{5} z_{6}+\frac{\gamma}{\beta} z_{3} z_{6} \\
\dot{z}_{2}=z_{4} z_{6} \\
\dot{z}_{3}=z_{6} \\
\dot{z}_{4}=f_{1} \\
\dot{z}_{5}=-\beta z_{5}+\beta\left(z_{4}+z_{1}\right) z_{6}+\gamma z_{6} \\
\dot{z}_{6}=f_{2}
\end{array} .\right.
$$

So far, system (3) is transformed to the form as system (4) by homeomorphic transformation, corresponding stabilization target is transformed as: design suitable control input $f_{1}, f_{2}$, 
make $z_{1}, z_{2}, z_{3}, z_{4}, z_{5}, z_{6}$ global asymptotic stabilization. To facilitate the analysis of system (4), it is written in the form of a cascade system:

$$
\begin{gathered}
\left\{\begin{array}{l}
\dot{z}_{1}=-\frac{\beta}{\alpha} z_{1}-\frac{\beta}{\alpha} z_{4}+z_{2} z_{6}-\frac{1}{\beta} z_{5} z_{6}+\frac{\gamma}{\beta} z_{3} z_{6} \\
\dot{z}_{5}=-\beta z_{5}+\beta\left(z_{4}+z_{1}\right) z_{6}+\gamma z_{6}
\end{array}\right. \\
\left\{\begin{array}{l}
\dot{z}_{2}=z_{4} z_{6} \\
\dot{z}_{3}=z_{6} \\
\dot{z}_{4}=f_{1} \\
\dot{z}_{6}=f_{2}
\end{array} .\right.
\end{gathered}
$$

\section{GLOBAL ASYMPTOTIC STABILIZATION ANALYZE}

First, here is the three lemmas required in the process of stabilization analysis: form:

Lemma 1[9]: the nonlinear cascade system is the following

$$
\begin{aligned}
& \sum_{1}: \dot{x}_{1}=f_{1}\left(t, x_{1}\right)+G(t, x) x_{2} \\
& \sum_{2}: \dot{x}_{2}=f_{2}\left(t . x_{2}, u\right)
\end{aligned}
$$

where, $x=\left[x_{1}, x_{2}\right]^{\mathrm{T}}$, function $f_{1}\left(t, x_{1}\right)$ is continuously differentiable on $\left(t, x_{1}\right), f_{2}\left(t, x_{2}, u\right), G(t, x)$ are local Lipschitz continuous in the interval. If meet the following conditions:

(1)system $\dot{x}_{1}=f_{1}\left(t, x_{1}\right)$ is global uniform asymptotic stabilized;

(2)exist control law $u$, make system $\sum_{2}: \dot{x}_{2}=f_{2}\left(t, x_{2}, u\right)$ global asymptotic stabilized;

(3)exist continuous function $\theta_{1}(\bullet): \mathbb{R}_{+} \rightarrow \mathbb{R}$ and $\theta_{2}(\bullet): \mathbb{R}_{+} \rightarrow \mathbb{R}$, make function $G(t, x)$ meet the conditions:

$$
\|G(t, x)\|_{2} \leq \theta_{1}\left(\left\|x_{2}\right\|_{2}\right)+\theta_{2}\left(\left\|x_{2}\right\|_{2}\right)\left\|x_{1}\right\|_{2}
$$

Then, the nonlinear cascade system is global uniform asymptotic stabilized.

Lemma 2[10]: if within a domain $D \subset R^{n}, f(t, x)$ and $\frac{\partial f}{\partial x}(t, x)$ is continuous in $[a, b] \times D$, then for $x, f$ is local Lipschitz continuous in $[a, b] \times D$.
Lemma 3(Barbalat Lemma): assume $x:[0, \infty) \rightarrow R$ is first order continuous derivative, when $t \rightarrow \infty$, exist finite limit, if $\dot{x}(t), t \in[0, \infty)$ is uniformly continuous, $\lim _{t \rightarrow \infty} \dot{x}(t)=0$.

This paper demonstrates the global asymptotic stability of system (4), by demonstrating system (4) (asymptotic system (5) and (6)) meet the conditions of lemma 1. First, setting $x_{1}=\left[z_{1}, z_{5}\right]^{\mathrm{T}}, \quad x_{2}=\left[z_{2}, z_{3}, z_{4}, z_{6}\right]^{\mathrm{T}}, \quad x=\left[x_{1}, x_{2}\right]^{\mathrm{T}}$, so equation (5) can be transformed as follow:

$$
\dot{x}_{1}=\left[\begin{array}{cc}
-\frac{\beta}{\alpha} & 0 \\
0 & -\beta
\end{array}\right] x_{1}+\left[\begin{array}{cccc}
z_{6} & \frac{\gamma}{\beta} z_{6} & -\frac{\beta}{\alpha} & -\frac{z_{5}}{\beta} \\
0 & 0 & \beta z_{6} & \beta z_{1}+\gamma
\end{array}\right] x_{2} \text {. }
$$

Setting

$$
\begin{aligned}
f_{1}\left(t, x_{1}\right) & =\left[\begin{array}{c}
-\frac{\beta}{\alpha} z_{1} \\
-\beta z_{5}
\end{array}\right]=\left[\begin{array}{cc}
-\frac{\beta}{\alpha} & 0 \\
0 & -\beta
\end{array}\right] x_{1} \\
G(t, x) & =\left[\begin{array}{cccc}
z_{6} & \frac{\gamma}{\beta} z_{6} & -\frac{\beta}{\alpha} & -\frac{z_{5}}{\beta} \\
0 & 0 & \beta z_{6} & \beta z_{1}+\gamma
\end{array}\right] \\
f_{2}\left(t, x_{2}, u\right) & =\left[\begin{array}{c}
z_{4} z_{6} \\
z_{6} \\
f_{1} \\
f_{2}
\end{array}\right]
\end{aligned}
$$

The First step: demonstrate system (5) meet the condition (1) of Lemma 1.

It can be known from the first equation of formula (7): $\dot{x}_{1}=f_{1}\left(t, x_{1}\right)=\left[\begin{array}{cc}-\frac{\beta}{\alpha} & 0 \\ 0 & -\beta\end{array}\right] x_{1}$

Obviously function $f_{1}\left(t, x_{1}\right)$ is continuously differentiable on $\left(t, x_{1}\right)$, and the system is linear time-invariant system, eigenvalues are $\lambda_{1}=-\frac{\beta}{\alpha}$ and $\lambda_{2}=-\beta$. By the definition of $\alpha$ and $\beta, \alpha$ and $\beta$ are all positive number, so, the two eigenvalues of system are strictly negative, system $\dot{x}_{1}=f_{1}\left(t, x_{1}\right)$ is global uniform asymptotic stabilized. Meet the condition (1) of Lemma 1 . Next, demonstrate the system meet the condition (3) of Lemma 1.

The second step: demonstrate system (5) meet the condition (3) of Lemma 1.

It can be known from the second equation of formula (7): 


$$
\begin{gathered}
G(t, x)=\left[\begin{array}{cccc}
z_{6} & \frac{\gamma}{\beta} z_{6} & -\frac{\beta}{\alpha} & -\frac{z_{5}}{\beta} \\
0 & 0 & \beta z_{6} & \beta z_{1}+\gamma
\end{array}\right] \\
G_{1}(t, x)=\left[\begin{array}{cccc}
z_{6} & \frac{\gamma}{\beta} z_{6} & -\frac{\beta}{\alpha} & 0 \\
0 & 0 & \beta z_{6} & \gamma
\end{array}\right] .
\end{gathered}
$$

Where:

By the Lemma 2, obviously can see that $G(t, x)$ is local Lipschitz continuous in the interval. The above formula can be broken into:

$$
\begin{aligned}
G(t, x) & =\left[\begin{array}{cccc}
z_{6} & \frac{\gamma}{\beta} z_{6} & -\frac{\beta}{\alpha} & -\frac{z_{5}}{\beta} \\
0 & 0 & \beta z_{6} & \beta z_{1}+\gamma
\end{array}\right] \\
& =\left[\begin{array}{cccc}
z_{6} & \frac{\gamma}{\beta} z_{6} & -\frac{\beta}{\alpha} & 0 \\
0 & 0 & \beta z_{6} & \gamma
\end{array}\right]+\left[\begin{array}{cccr}
0 & 0 & 0 & -\frac{z_{5}}{\beta} \\
0 & 0 & 0 & \beta z_{1}
\end{array}\right] .
\end{aligned}
$$

according to the property of norm, it can be known:

$$
\left\|G_{1}(t, x)\right\|_{2} \leq\left(\beta+1+\left|\frac{\gamma}{\beta}\right|\right)\left\|z_{6}\right\|_{2}+\frac{\beta}{\alpha}+|\gamma|
$$

And because of $\left\|z_{6}\right\|_{2} \leq\left\|x_{2}\right\|_{2}$, inequation (8) can be transformed into:

$$
\left\|G_{1}(t, x)\right\|_{2} \leq\left(\beta+1+\left|\frac{\gamma}{\beta}\right|\right)\left\|x_{2}\right\|_{2}+\frac{\beta}{\alpha}+|\gamma|=\theta_{1}\left(\left\|x_{2}\right\|_{2}\right)
$$

where, $\theta_{1}$ is a function of $\left\|x_{2}\right\|_{2}$.

Similarly, define: $G_{2}(t, x)=\left[\begin{array}{rrrr}0 & 0 & 0 & -\frac{z_{5}}{\beta} \\ 0 & 0 & 0 & \beta z_{1}\end{array}\right]$

According to the property of norm, it can be known:

$$
\left\|G_{2}(t, x)\right\|_{2} \leq\left(\frac{1}{\beta}+\beta\right) \sqrt{z_{1}^{2}+z_{5}^{2}}=\left(\frac{1}{\beta}+\beta\right)\left\|x_{1}\right\|_{2}=\theta_{2}\left(\left\|x_{2}\right\|_{2}\right)\left\|x_{1}\right\|_{2} .
$$

where, $\theta_{1}$ is a function of $\left\|x_{2}\right\|_{2}$.

So, according to the property of norm and formula (9) and (10), obtain:

$$
\|G(t, x)\|_{2} \leq\left\|G_{1}(t, x)\right\|_{2}+\left\|G_{2}(t, x)\right\|_{2} \leq \theta_{1}\left(\left\|x_{2}\right\|_{2}\right)+\theta_{2}\left(\left\|x_{2}\right\|_{2}\right)\left\|x_{1}\right\|_{2}
$$

Therefore, meet the condition (3) of Lemma 1. In order to illustrate the system meet the condition (2) of Lemma 1 better, design the stabilization controller of system (6) firstly.

\section{CONTROLler DESIGN}

First, system (6) can be divided into two subsystems as follow:

$$
\begin{gathered}
\sum_{21}:\left[\begin{array}{c}
\dot{z}_{2} \\
\dot{z}_{3} \\
\dot{z}_{4}
\end{array}\right]=\left[\begin{array}{c}
z_{4} z_{6} \\
z_{6} \\
f_{1}
\end{array}\right] . \\
\sum_{22}:\left[\dot{z}_{6}\right]=\left[f_{2}\right] .
\end{gathered}
$$

The first part: in this part, design the control input $f_{1}$ by subsystem (11) of the USV stabilization system.

Assume virtual input $z_{6}=\Gamma$, and subsystem (11) is transformed into the following type:

$$
\sum_{21}:\left[\begin{array}{c}
\dot{z}_{2} \\
\dot{z}_{3} \\
\dot{z}_{4}
\end{array}\right]=\left[\begin{array}{c}
z_{4} \Gamma \\
\Gamma \\
f_{1}
\end{array}\right] \text {. }
$$

where, $z_{2}, z_{3}, z_{4}$ are state variable, $f_{1}$ and $\Gamma$ are input variable. For system (13), select Lyapunov function as follow:

$$
V_{1}=\frac{1}{2} k_{2} z_{2}^{2}+\frac{1}{2} z_{4}^{2} \geq 0
$$

Calculate the derivative of (14), and bring the formulas of (13) in, obtain:

$$
\dot{V}_{1}=k_{2} z_{2} \dot{z}_{2}+z_{4} \dot{z}_{4}=k_{2} z_{2} z_{4} \Gamma+z_{4} f_{1}=z_{4}\left(k_{2} z_{2} \Gamma+f_{1}\right)
$$

For formula (15), select the control law as follow:

$$
f_{1}=-k_{4} z_{4}-k_{2} z_{2} \Gamma
$$

Therefore

$$
\dot{V}_{1}=z_{4}\left(k_{2} z_{2} \Gamma+f_{1}\right)=-k_{4} z_{4}^{2} \leq 0
$$

Formula (14) and (17) show that $V_{1}$ is nonincremental, and $V_{1}$ will converge to a limit $V_{\text {lim }}(\geq 0)$, so get that $\lim _{t \rightarrow \infty} z_{4}=0$, and $z_{2}$ is bounded.

In order to ensure $Z_{2}$ converges to zero, this paper select 


$$
\Gamma=\dot{z}_{3}=-k_{3} z_{3}+\lambda z_{2}
$$

where, $\lambda$ is bounded.

Demonstrate $\Gamma$ converges to zero by paradox: assume $\Gamma$ does not converge to zero, it has been demonstrated $Z_{4}$ converges to zero already, and obtain that $z_{2} \Gamma$ converges to zero by Lemma 3 , thus deduced $z_{2}$ converges to zero, combining with (18) know that $z_{3}$ converges to zero too, then obtain that $\Gamma$ converges to zero, it is inconsistent with the assumed condition. Thus demonstrate that $\Gamma$ converges to zero.

Select $\lambda=-\sin t$, then

$$
\begin{gathered}
\Gamma=-k_{3} z_{3}+\lambda z_{2}=-k_{3} z_{3}-z_{2} \sin t \\
\dot{\Gamma}=-k_{3} \dot{z}_{3}-\dot{z}_{2} \sin t-z_{2} \cos t=-k_{3} \Gamma-z_{4} \Gamma \sin t-z_{2} \cos t
\end{gathered}
$$

Where, $k_{3} \Gamma$ and $z_{4} \Gamma \sin t$ converge to zero, $z_{2} \cos t$ is uniformly continuous, combining with Lemma $3, z_{2} \cos t$ converges to zero, so $z_{2} \cos ^{2} t$ converges to zero too. Calculate the derivative of $z_{2}$ cost , and combine with Lemma $3, z_{2} \sin t$ converges to zero in the same way, so $z_{2} \sin ^{2} t$ converges to zero, and $z_{2}=z_{2} \cos ^{2} t+z_{2} \sin ^{2} t$ converges to zero. Through $\Gamma=-k_{3} z_{3}+\lambda z_{2}=-k_{3} z_{3}-z_{2} \sin t \quad, \quad z_{3}$ converges to zero too. Similar situation, when $\lambda= \pm \sin t$ or $\lambda= \pm \cos t$, the control law in the follow can make system (13) global asymptotic stabilized.

$$
\left\{\begin{array}{l}
\Gamma=\dot{z}_{3}=-k_{3} z_{3}+\lambda z_{2} \\
f_{1}=-k_{4} z_{4}-k_{2} z_{2} \Gamma
\end{array} .\right.
$$

The first part: in this part, design the control input $f_{2}$ by stabilizing the USV system (6), then obtain the global stabilization control law of USV.

Assume $\Theta=z_{6}-\Gamma$, then $z_{6}=\Theta+\Gamma$. System (6) can be written as follow:

$$
\left\{\begin{array}{l}
\dot{z}_{2}=z_{4}(\Theta+\Gamma) \\
\dot{z}_{3}=\Theta+\Gamma \\
\dot{z}_{4}=-k_{2} z_{2} \Gamma-k_{4} z_{4} \\
\dot{z}_{6}=f_{2}
\end{array}\right.
$$

Select the Lyapunov function of system (20) as follow:

$$
V_{2}=\frac{1}{2} k_{2} z_{2}^{2}+\frac{1}{2} z_{4}^{2}+\frac{1}{2} \Theta^{2} \geq 0
$$

Calculate the derivative of formula (21), get

$$
\begin{aligned}
\dot{V}_{2} & =k_{2} z_{2} \dot{z}_{2}+z_{4} \dot{z}_{4}+\Theta \dot{\Theta} \\
& =k_{2} z_{2} z_{4}(\Theta+\Gamma)+z_{4}\left(-k_{2} z_{2} \Gamma-k_{4} z_{4}\right)+\Theta\left(\dot{z}_{6}-\dot{\Gamma}\right) \\
& =-k_{4} z_{4}^{2}+k_{2} z_{2} z_{4} \Theta+\Theta\left(f_{2}-\dot{\Gamma}\right) \\
& =-k_{4} z_{4}^{2}+\Theta\left(f_{2}-\dot{\Gamma}+k_{2} z_{2} z_{4}\right)
\end{aligned}
$$

Based on formula (22), select the control law as follow:

$$
f_{2}=\dot{\Gamma}-k_{2} z_{2} z_{4}-k_{6} \Theta=\dot{\Gamma}-k_{2} z_{2} z_{4}-k_{6}\left(z_{6}-\Gamma\right) .
$$

bring the formulas of (23) in formula (22),get:

$$
\dot{V}_{2}=-k_{4} z_{4}^{2}+\Theta\left(-k_{6} \Theta\right)=-k_{4} z_{4}^{2}-k_{6} \Theta^{2} \leq 0
$$

Formula (21) and (24) show that $V_{2}$ is nonincremental, and $V_{2}$ will converge to a $\operatorname{limit} V_{2 \lim }(\geq 0)$, get that $\lim _{t \rightarrow \infty} \Theta=0$, and due $\lim _{t \rightarrow \infty} \Gamma=0$, so $\lim _{t \rightarrow \infty} z_{6}=0$.

Comprehensive the above contents, for system (6), the control law in the follow is able to make $Z_{2}, Z_{3}, Z_{4}, z_{6}$ global uniform asymptotic stabilized.

Now back to the third step of the global asymptotic stabilization analyze in the third section: demonstrate system (6) meet condition (2) of Lemma 1.

Through the process of design system (6) stabilization controller, obviously show that the system meet condition (2) of Lemma 1. Through Lemma 2, obviously $f_{2}\left(t, x_{2}, u\right)$ is locally Lipschitz continuous.

Through a set of demonstrating process above, it can be seen that the underactuated USV system (4),that is system (5) and system (6), meet all the conditions of Lemma 1, so system (4) is global uniform asymptotic stabilized, and the control law is formula (33).

\section{Simulation RESUlts}

Using the control law designed above doing USV stabilization control simulation experiment, the model parameters of the USV is[11]: $m_{11}=1.1274, m_{22}=1.8902$, $m_{33}=0.1278, m_{23}=-0.0744, m_{32}=-0.0744, d_{11}=0.0358$, $d_{22}=0.1183, d_{33}=0.0308, d_{23}=-0.0124, d_{32}=-0.0041$. The initial state of USV is set to: $x(0)=-1, y(0)=-1$, $\varphi(0)=\frac{\pi}{2}, u(0)=0, v(0)=0, r(0)=0$. Parameter selection 
for the control: $k_{2}=1.5, k_{3}=0.8, k_{4}=1.12, k_{6}=1$, $\lambda=\sin t$. The simulation results are shown in the figure below:

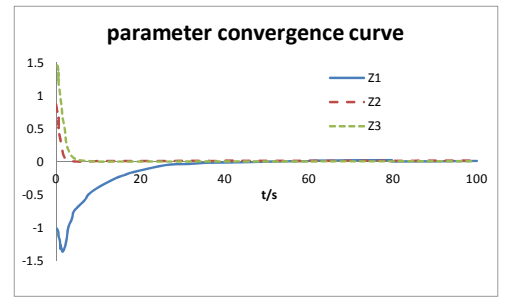

FIGURE I. CONVERGENCE CURVE OF $\quad z_{1}, z_{2}, z_{3}$

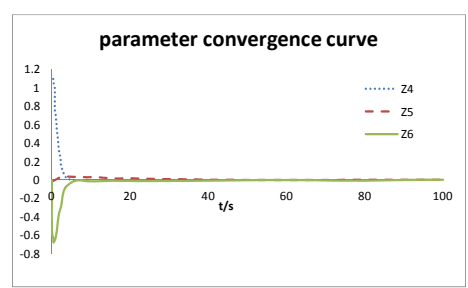

FIGURE II. CONVERGENCE CURVE OF $z_{4}, z_{5}, z_{6}$

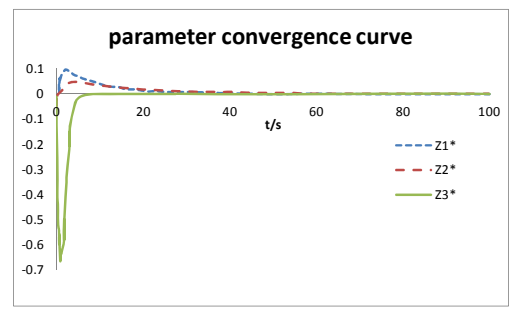

FIGURE III. CONVERGENCE CURVE OF $z_{1}^{*}, z_{2}^{*}, z_{3}^{*}$

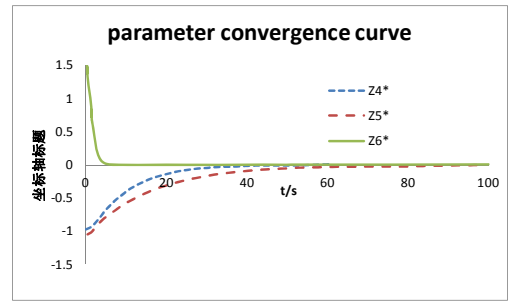

FIGURE IV. CONVERGENCE CURVE OF $z_{4}^{*}, z_{5}^{*}, z_{6}^{*}$

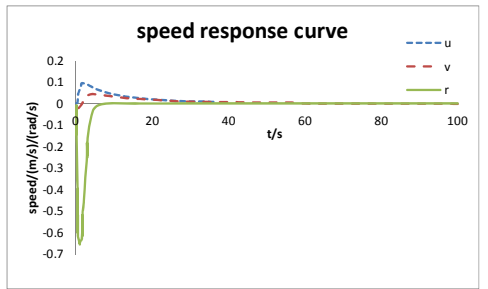

FIGURE V. CONVERGENCE CURVE OF $u, v, r$

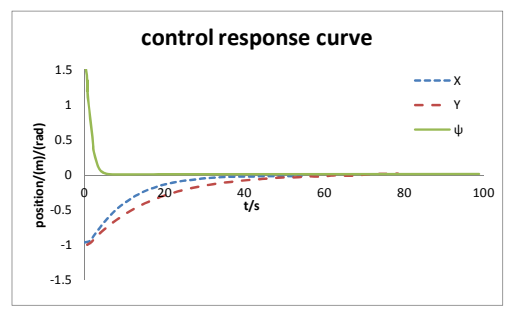

FIGURE VI. CONVERGENCE CURVE OF $x, y, \varphi$

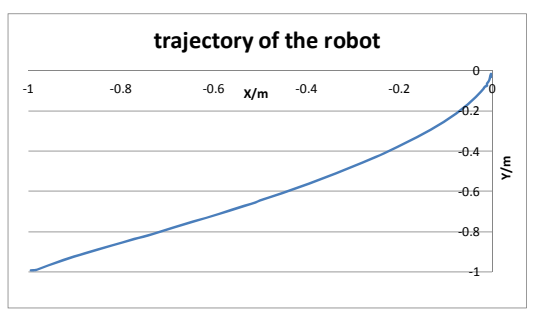

FIGURE VII. TRAJECTORY CURVE OF USVFIG.

In the figure above, Figure I and Figure II show convergence curves of state variables $z_{1}, z_{2}, z_{3}, z_{4}, z_{5}, z_{6}$ of system (4) in the simulation process, Figure III and Figure IV show convergence curves of state variables $z_{1}^{*}, z_{2}^{*}, z_{3}^{*}, z_{4}^{*}, z_{5}^{*}, z_{6}^{*}$ of system (3) in the simulation process. Figure V and Figure VI show convergence curves of the speed variables ${ }^{u, v, r}$ and position variables $x, y, \varphi$ of original system (1) and (2) in the USV stabilization control simulation process, Figure VII show the trajectory curve of USV in the stabilization process. Through a series of simulation results above, it can be seen the original incomplete symmetrical underactuated USV system (1) and (2), and the transformed system (3) and (4), all the state variables of them converge to zero uniformly, this means that these three systems are all global uniform asymptotic stabilized. The simulation results demonstrate the effectiveness of the twice global diffeomorphism transformation and the control law designed in this article.

\section{ConClusions}

This paper study the stabilization control of underactuated USV, while the USV model is incomplete symmetrical, through performing global diffeomorphism transformation twice, obtain the form of cascade systems, design the control law based on Backstepping, make the system achieve global asymptotic stabilized, and analyze the stability of the stabilization control. Finally, demonstrate the method proposed and controller designed in this paper can make the incomplete symmetrical underactuated USV realize global asymptotic stabilization.

\section{ACKNOWLEDGEMENT}

We gratefully acknowledge the support of the National Natural Science Foundation of China (51609054).

\section{REFERENCE}

[1] Reyhanoglu M. Control and stabilization of an underactuated surface vessel[C]. Proceedings of the 35th IEEE Conference on Decision and Control, Kobe, Japan, 1996: 2371-2376

[2] Ghommam J, Mnif F, Benali A, Derbel N. Asymptotic backstepping stabilization of an underactuated surface vessel[J]. IEEE Transactions on Control Systems Technology. 2006, 14(6): 1150-1157 
[3] LIAO Yulei, PANG Yongjie, ZHANG Tiedong. Global K-exponential stabilization of underactuated automonous surface vessels by a smooth time-varying feedback control [J]. Journal of Harbin Engineering University, 2011, 32(4): 417-422.)

[4] Ma Baoli. Global k-exponential asymptotic stabilization of underactuated surface vessels [J]. Systems \& Control Letters. 2009, 58: 191-201P

[5] LIU Yang, GUO Chen, LIU Yu. Discontinuous varying parameters stabilization control for underactuated surface vessel motion [J]. Journal of Dalian Maritime University, 2009, 35(1): 9-12.)

[6] Kristin Y Pettersen, Thor I Fossen. Underactuated dynamic positioning of a ship-experimental results [J]. IEEE Transactions on Control Systems Technology, 2000, 8(5): 856-863

[7] Frdric Mazenc, Kristin Pettersen, Henk Nijmeijer. Global uniform asymptotic stabilization of an underactuated surface vessel [J]. IEEE Transactions on Automatic Control, 2002, 47(10): 1759-1762.

[8] Thor I. Fossen. Guidance and Control of Ocean Vehicles [M] University of Trondheim, Norway, 1994.

[9] E. Lefeber. Tracking control of nonlinear mechanical systems. Universiteit Twente Ph.D. thesis. 2000

[10] Hassan K. Khalil. Nonlinear Systems, Third Edition [M]. Beijing: Publishing House of Electronics Industry. 2011

[11] Do K D, Pan J. Global tracking control of underactuated ships with off-diagonal terms $[\mathrm{C}]$. Proceeding of 42nd IEEE Conference on Decision and Control. Hawaii, 2003: 1250-1255. 